\title{
The coupling between gaze behavior and opponent kinematics during anticipation of badminton shots
}

\author{
David Alder $^{\mathrm{a}, *}$, Paul R. Ford ${ }^{\mathrm{a}, 1}$, Joe Causer ${ }^{\mathrm{a}, 2}$, A. Mark Williams ${ }^{\mathrm{b}, 3}$ \\ ${ }^{a}$ Liverpool John Moores University, Tom Reilly Building, Byrom Street, Liverpool L3 3AF, United Kingdom \\ ${ }^{\mathrm{b}}$ Brunel University, Heinz Wolff Building, HW201, Uxbridge UB8 3PH, United Kingdom
}

\section{A R T I C L E I N F O}

\section{Article history:}

Available online 16 September 2014

\section{PsycINFO classification:}

2323

Keywords:

Visual perception

Biomechanics

Expert performance

Decision making

Eye movements

\begin{abstract}
A B S T R A C T
Purpose: We examined links between the kinematics of an opponent's actions and the visual search behaviors of badminton players responding to those actions.

Method: A kinematic analysis of international standard badminton players $(n=4)$ was undertaken as they completed a range of serves. Video of these players serving was used to create a life-size temporal occlusion test to measure anticipation responses. Expert $(n=8)$ and novice $(n=8)$ badminton players anticipated serve location while wearing an eye movement registration system.

Results: During the execution phase of the opponent's movement, the kinematic analysis showed between-shot differences in distance traveled and peak acceleration at the shoulder, elbow, wrist and racket. Experts were more accurate at responding to the serves compared to novice players. Expert players fixated on the kinematic locations that were most discriminating between serve types more frequently and for a longer duration compared to novice players. Moreover, players were generally more accurate at responding to serves when they fixated vision upon the discriminating arm and racket kinematics.

Conclusions: Findings extend previous literature by providing empirical evidence that expert athletes' visual search behaviors
\end{abstract}

\footnotetext{
* Corresponding author. Tel.: +44 07814843933; fax: +44 01519046284.

E-mail addresses: d.alder@2008.ljmu.ac.uk (D. Alder), p.ford@ljmu.ac.uk (P.R. Ford), j.causer@ljmu.ac.uk (J. Causer), mark. williams@brunel.ac.uk (A.M. Williams).

1 Tel.: +44 0151904 6246; fax: +4401519046284.

2 Tel./fax: +4401519046284.

${ }^{3}$ Tel.: +44 01895 267605; fax: +44 01519046284 .
} 
and anticipatory responses are inextricably linked to the opponent action being observed.

(C) 2014 Elsevier B.V. All rights reserved.

\section{Introduction}

Expert athletic performance consists of many perceptual, cognitive and motor elements (Causer, Janelle, Vickers, \& Williams, 2012). A key element in elite sport is the ability to anticipate opponent actions prior to their completion (Williams, Ford, Eccles, \& Ward, 2011). Expert athletes are able to anticipate opponent actions by using vision to extract information from their movements prior to a key event in the action, such as ball-racket or ball-foot contact (Abernethy \& Russell, 1987; Abernethy, Zawi, \& Jackson, 2008; Savelsbergh, Williams, Van der Kamp, \& Ward, 2002; Williams, Ward, Knowles, \& Smeeton, 2002). However, contradictory findings have emerged in the literature as to the kinematic information that athletes should allocate visual attention to when making anticipation judgments. These conflicting findings are found between and within researchers examining the visual fixations of athletes during anticipation judgments and those examining where the kinematic differences between actions occur, probably because in both cases neither quantifies the other. In this study, we examine, for the first time in the literature, the coupling between the kinematics of opponent actions and the associated visual search behaviors of athletes who are attempting to anticipate those actions.

Previously, researchers have investigated the kinematic differences between actions (Huys, Smeeton, Hodges, Beek, \& Williams, 2008), while others have separately examined the kinematic information that athletes fixate vision upon during anticipation (Williams et al., 2002). Huys et al. (2008) used principal component analysis (PCA) to investigate the kinematic patterns that discriminated between forehand tennis strokes to four locations in the opponent's court. The shots varied in direction and depth by being 'cross court' and 'inside-out' to both short and long areas of the court. The kinematics differed between shots as a function of direction, but not shot depth. The authors found that kinematic differences between shots to the left or right occurred at locations across the whole body. In a similar study, Bourne, Bennett, Hayes, and Williams (2011) used PCA to examine handball shots directed to each of the four corners of the goal. In contrast to Huys et al. (2008), kinematic patterns between shots to the four different locations were not significantly different, suggesting that between-shot differences may be subtle and related to changes in refined hand kinematics, which were not measured. Differences between actions in a sport are clear when they are somewhat exaggerated and/or occur across all of the body, such as the 'cross court' versus 'inside-out' tennis shots examined by Huys et al. (2008). As such, visual search research using tennis shots (Ward, Williams, \& Bennett, 2002; Williams et al., 2002) has shown that the locations of fixations tend to be distributed across a number of central regions of the body (e.g., head, trunk), which perhaps act as an 'anchor point' of fixation that enables peripheral vision to pick up the kinematic differences in the multiple locations (Ripoll, Kerlirzin, Stein, \& Reine, 1995). However, when the differences between actions are subtler, the discriminating differences between shots occur in fewer and often distal body locations (e.g., hands in Bourne et al., 2011). For example, research examining visual search behaviors during badminton shots shows the fixations are located on specific distal areas of the body (arm, wrist, and racket in Abernethy and Russell (1987), because kinematic differences between badminton shot types are hypothesized to be subtle and occurring in these distal areas. Variation in the amount of kinematic locations or information available that differentiate between and within actions might lead to related variation in the locations of visual search fixations used to extract this information. The evidence suggests that the kinematics of the opponents' action and the visual search behavior of the athlete anticipating those actions are inextricably linked, which implies that they should not be examined in isolation. 
The effectiveness of anticipation judgments may be dependent upon whether the athlete fixates on the correct kinematic locations where the differences between actions are occurring (Savelsbergh, Van der Kamp, Williams, \& Ward, 2005). Alternatively, incorrect anticipation might occur when athletes fixate on the correct kinematic differences but fail to recognize or use this information. However, researchers have measured the visual search behaviors used in anticipation without differentiating the effectiveness of the judgment. There is a need to examine visual search behaviors as a function of anticipatory judgment outcome to reveal the underlying reasons for success and errors. Moreover, some researchers have established that athletes change the areas they fixate vision upon across the on-going movement (Abernethy \& Russell, 1987; Kim \& Lee, 2006; Savelsbergh et al., 2002), since the amount or type of kinematic information emanating from different areas of the opponent's body changes across the movement. Researchers have generally not examined this temporal component of visual search behavior (for exceptions, see Abernethy \& Russell, 1987; Kim \& Lee, 2006; Savelsbergh et al., 2002) despite the need to know "when" exactly athletes fixate upon certain kinematic information as it unfolds across the observed action.

The aim in this study was to examine kinematic differences between types of badminton serves and quantify whether players fixate vision upon that discriminating kinematic information during successful anticipation judgments. In the first section of the paper, a kinematic analysis of expert players executing badminton serves was conducted. In the second section, a new set of expert and novice players took part in a temporal occlusion test in which they were required to anticipate the landing locations of those serves while their visual search behaviors were recorded. It was expected that the kinematic analysis would reveal between shot-type differences in the arm, wrist, and racket regions (Abernethy \& Russell, 1987). It was predicted that expert players would fixate vision upon these between-shot differences more frequently compared to the novice players. The visual fixations of expert players were predicted to be based upon the kinematic differences between shots during the phases of the movement in which they emerge on more trials compared to the novice players. The between-group differences in visual search strategies were expected to be associated with greater accuracy of anticipation judgments for the expert compared to novice players. Moreover, when fixating on areas of the opponent's body that discriminate shot type at the time in which they become discriminatory, both groups were predicted to have significantly more successful anticipation judgments compared to when fixating on other areas of the visual display.

\section{Method}

\subsection{Participants}

Participants were four expert badminton players $(M=25.6$ years of age, $S D=2.3)$ from Great Britain (GB) who were on the Team GB World Class Development Programme for athletes with the potential to medal at international events. They were taking part in $20 \mathrm{~h}$ a week of badminton specific practice and they had played badminton regularly for over 10 years, participating in numerous international competitions. Each participant provided informed consent prior to the study. Full approval was provided by the local ethics committee.

\subsection{Procedure}

Each participant had a set of passive reflective markers positioned on numerous anatomical locations in order to replicate the University of Western Australia (UWA) full body marker set (Dempsey, Lloyd, Elliott, Steele, \& Munro, 2009). Two further reflective markers were positioned on the racket head at approximately the $10.00 \mathrm{~h}$ (termed from here as "racket 1 ") and $02.00 \mathrm{~h}$ (termed from here as "racket 2") locations of a "clock face" where the racket head/handle intersection would be $06.00 \mathrm{~h}$. A marker was also placed on the cork of the shuttle. The right service box of a badminton court was then encircled by four Motion Capture cameras (Qualysis Pro-Reflex MCO 1000, Sweden) synchronized with the Qualysis Motion Capture System (Version 1.10.2xx, Sweden) to record the participant's movements. The participants were required to perform 10 short and 10 long serves to the 
corresponding service box on the opposite side of the court as per a traditional badminton serve. A panel of three Olympic badminton coaches chose the serve in a doubles match as the shot to be examined in this study. They stated that in elite badminton doubles matches, anticipating the serve is one of the most important attributes a player needs to possess, with the majority of points being won by the team that gain the advantage at the return of serve.

\subsection{Data analysis}

A standard biomechanical analysis was conducted on each serve, consisting of movement duration, total distance traveled, peak acceleration and peak velocity (Lees, 2002). All dependent variables were recorded from the start of the serve, defined as when the server's feet were set, until the end of serve, defined as shuttle/racket contact. Coaching literature from badminton indicated that players anticipate shot depth during the early stages of the server's movement, whereas shot direction (e.g., left, right) is recognized during initial shuttle flight (Badminton Association of England, Level 1: Assistant coach training manual, 2005). Therefore, all dependent variables were calculated as a function of depth (long, short). Additionally, after initial inspection of the serves, the action was separated into two distinct phases termed the preparation and execution phases. The preparation phase was defined as from the video frame in which the server's feet were set to the frame in which the racket and shuttle were set prior to their final forward motion. The execution phase was defined as from the frame containing the set point of the racket and shuttle up until that containing racket-shuttle contact.

First, movement duration was calculated as a function of phase. Second, total distance traveled was calculated for each marker for each of the serves as a function of movement phase. Total distance covered was calculated by summing the movement path distances of a marker across an action. Displacement data were not calculated as a variable within this study as it only provides the length of the line between the start and end point of the marker and, therefore, has the potential to provide a null value. Total distance covered for each marker between short and long serves in each phase was analyzed using separate paired $t$-tests on each marker. Only the markers that differentiated short from long serves for distance covered were selected for further analysis, as the other markers were deemed not part of the discriminating information indicating the length of the shot to the server's opponent. Third, peak acceleration was calculated and analyzed using separate paired $t$-tests across both phases for only those markers that were differentiated for their total distance covered between long and short serves. ${ }^{4}$ In order to limit the potential inflation of type- 1 errors through multiple $t$-tests, each alpha value was adjusted using the Bonferroni correction method. Updated alpha values are reported throughout.

\section{Results}

The average movement duration was $4550 \mathrm{~ms}(\mathrm{SD}=0.65)$ consisting of the preparation $(M=3400 \mathrm{~ms}, \mathrm{SD}=500)$ and execution phase $(M=1900 \mathrm{~ms}, \mathrm{SD}=320)$. Total distance covered was significantly greater in seven out of the 28 markers in one of the movement phases at least for long compared to short serves. The distance traveled for these seven markers and the statistical results for this dependent variable are shown in Table 1 for the preparation phase of the movement and Table 2 for the execution phase. No differences were found in the preparation phases for distance traveled in any of the markers. However, in the execution phase, total distance traveled was greater in long compared to short serves in the right elbow, medial right wrist, 3rd carpal on right wrist, racket 1 , racket 2, lateral right wrist and right shoulder (all $p<.01$ ). These seven kinematic markers and locations were subjected to further analysis.

In the preparation phase, peak acceleration was greater in long compared to short serves in the 3rd carpal on right wrist $(p<.01)$ and racket $2(p<.01)$. There were no significant differences found for peak acceleration for the other five markers. Table 3 shows the peak acceleration of the seven markers in the execution phase of the movement, as well as the statistical results for this dependent variable.

\footnotetext{
${ }^{4}$ Peak velocity was also calculated for the seven markers that differentiated short from long serves. The data did not differ to the peak acceleration data and was, therefore, not included in order that the paper remain concise.
} 
Table 1

Means and standard deviations of distance traveled in $\mathrm{mm}$ for the markers of interest in preparation phase of the serve.

\begin{tabular}{llll}
\hline Marker Location & Short & Long & $t$ value \\
\hline Right shoulder & $156.04(34.88)$ & $137.07(36.74)$ & $t_{3}=0.66, p=.56$ \\
Right elbow & $156.16(43.28)$ & $167.68(44.79)$ & $t_{3}=-9.87, p=.57$ \\
Lateral right wrist & $376.81(81.76)$ & $363.27(5.89)$ & $t_{3}=0.33, p=.76$ \\
Medial right wrist & $296.70(199.30)$ & $337.33(147.67)$ & $t_{3}=-0.87, p=.49$ \\
3rd Carpal on right wrist & $291.40(11.69)$ & $370.42(165.98)$ & $t_{3}=-0.94, p=.42$ \\
Racket 1 & $543.39(142.25)$ & $645.54(11.69)$ & $t_{3}=-1.34, p=.27$ \\
Racket 2 & $591.35(169.49)$ & $671.46(22.32)$ & $t_{3}=-0.92, p=.42$ \\
\hline
\end{tabular}

Table 2

Means and standard deviations of distance traveled in $\mathrm{mm}$ for the markers of interest in execution phase of the serve.

\begin{tabular}{llll}
\hline Marker location & Short & Long & $t$ value \\
\hline Right shoulder & $22.55(6.37)$ & $25.63(13.07)$ & $t_{3}=-0.46, p<.01$ \\
Right elbow & $21.29(5.90)$ & $33.75(4.35)$ & $t_{3}=-5.28, p<.01$ \\
Lateral right wrist & $20.47(0.47)$ & $49.54(0.81)$ & $t_{3}=173.93, p<.01$ \\
Medial right wrist & $22.97(6.14)$ & $37.80(10.27)$ & $t_{3}=-2.47, p<.07$ \\
3rd Carpal on right wrist & $21.93(0.88)$ & $53.07(10.50)$ & $t_{3}=-5.80, p<.01$ \\
Racket 1 & $31.52(0.60)$ & $48.59(0.88)$ & $t_{3}=-60.66, p<.01$ \\
Racket 2 & $33.22(22.41)$ & $50.54(11.21)$ & $t_{3}=-61.51, p<.01$ \\
\hline
\end{tabular}

Table 3

Means and standard deviations of peak acceleration in $\mathrm{mm} / \mathrm{s}$ for the markers of interest in execution phase of the serve.

\begin{tabular}{llll}
\hline Marker location & Short & Long & $t$ value \\
\hline Right shoulder & $804.17(3.40)$ & $6025.19(5.84)$ & $t_{3}=-4829.23, p<.01$ \\
Right elbow & $1343.52(84.27)$ & $1733.22(40.83)$ & $t_{3}=-320.16, p<.01$ \\
Lateral right wrist & $4879.65(3.32)$ & $10363.21(5.68)$ & $t_{3}=-4625.98, p<.01$ \\
Medial right wrist & $1577.61(27.41)$ & $4786.50(38.92)$ & $t_{3}=-2354.25, p<.01$ \\
3rd Carpal on right wrist & $4995.08(5.27)$ & $5365.38(2.47)$ & $t_{3}=-181.79, p=.03$ \\
Racket 1 & $6100.24(4.08)$ & $16162.76(58.73)$ & $t_{3}=-8266.70, p<.04$ \\
Racket 2 & $2884.49(3.41)$ & $9811.04(41.11)$ & $t_{3}=-5690.49, p=.01$ \\
\hline
\end{tabular}

In the execution phase, peak acceleration was greater in long compared to short serves in all seven markers. It differentiated short from long serves in the right shoulder, right elbow, medial right wrist, lateral right wrist, 3 rd carpal on right wrist, racket 1 and racket 2 (all $p<.01$ ).

\section{Method}

\subsection{Participants}

Participants were eight expert $(M=28.9$ years of age, $\mathrm{SD}=3.1)$ and eight novice $(M=18.5$ years of age, $\mathrm{SD}=1.1$ ) badminton players. Expert participants were on the Team GB World Class Development Programme for athletes who have potential to medal at World or Olympic events. The expert participants were taking part in $20 \mathrm{~h}$ a week of badminton specific practice and had played badminton regularly for over 10 years, participating in international competitions. In contrast, novice participants were individuals who had not taken part in any structured badminton training or competition. Each participant provided informed consent prior to the study. Full approval was provided by the local ethics committee. 


\subsection{Video recording and film construction}

A domain-specific representative task was created for this study using life-sized video footage of players completing a variety of serves from the perspective of an opponent in a doubles match. To create the task, the four international badminton players from the first section of the paper were videotaped completing a range of serves. A high-definition (HD) video camera (Canon XHA1S; Tokyo, Japan) was positioned two meters away from the net at eye level (1.7 meters) so as to provide the most realistic representation of the opponent's view. The players completed long and short serves to three different directions/locations on the opponent's court. The locations were the tee (the point at which the center line meets the service line or back tramline), centre of service box, and wide to the left and right service box. The six areas of the court were identified by the same panel of Olympic coaches as being the most commonly used during a badminton serve in a doubles match. Although the primary independent variable was shot depth, three shot directions were included in the video (central, left and right), so as to increase the difficulty of the task, lowering the level of chance on the test from 50\% (long, short) to 17\% (six landing locations). During filming, another individual was positioned on court who acted as the doubles partner for the server. Both the server and their partner could be viewed on the video. Each of the servers performed three shots to each of the six locations, making a total of 18 serves per server, and 72 serves in total.

Serves were edited (Adobe Premier Pro Editing Software, Version CS5, San Jose, USA) into video clips to be used in a test film. Each video clip began with a black screen for $2000 \mathrm{~ms}$ containing white text. Since the experiment was to take place on a badminton court, the text informed the participant to stand in the left or right service box so as to receive the on screen serve. At $2000 \mathrm{~ms}$, another black screen showed white text of a "3, 2, 1" countdown that lasted $2000 \mathrm{~ms}$. At $4000 \mathrm{~ms}$, a still picture of the initial video frame of the service action was shown for $500 \mathrm{~ms}$. At 4,500 ms, the video began with the initial still frame containing the start of the trial for $500 \mathrm{~ms}$ and the beginning of the video of the movement/preparation phase started at $5000 \mathrm{~ms}$ approximately. The video ran for approximately $4500 \mathrm{~ms}$. The clips ended with a black screen that occluded for $3000 \mathrm{~ms}$. Each clip was occluded at one of three time points: $40 \mathrm{~ms}$ pre racket/shuttle contact; at racket/shuttle contact; and $40 \mathrm{~ms}$ post racket/shuttle contact. The trials were presented in a random order across the test film, but kept in the same order for each participant.

\subsection{Procedure}

Prior to the experiment, participants received instruction about the rationale and protocol of the study. A mobile eye-tracking system (ASL MobileEye, Bedford, USA) was used to record gaze behaviors. The mobile eye-tracking system is a head-mounted, monocular eye-tracking system that computes point of gaze within a scene through calculation of the vector between the participant's pupil and cornea. The calibration consisted of participants fixating to six pre-determined location points on a still image of one of the trials (server's head, racket head, left foot, shuttle, non-server's head and racket head). During calibration, participants were instructed to adopt the typical stance used when returning serve.

Testing took place on a full size international standard badminton court. The test film was back projected life-size onto a two-dimensional screen (size $2.74 \mathrm{~m}$ high $\times 3.66 \mathrm{~m}$ wide, Draper, USA). The screen was positioned on the opposite side of the court at $1.98 \mathrm{~m}$ from where the net would be in a position that provided the most representative view of the serves. Participants were required to start each trial on either the left or right hand side of the service area, as they would do in a normal badminton match. The start locations were clearly marked on the floor with an " $X$ " using tape. Participants were required to physically carry out a return shot as they would do in a match and to provide verbal confirmation as to the end location of the serve. The experimenter hand notated the verbal response of the participant during the experiment. The physical return shot was not recorded as a dependent variable, but was used to increase the fidelity of the task. A time limit was set for the verbalized and movement response to occur at $3000 \mathrm{~ms}$ after the video occlusion, which approximately corresponded to the moment when the shuttlecock would reach the ground on film and become 
unplayable. Any responses that occurred after the time limit were deemed incorrect. At the $3000 \mathrm{~ms}$ time limit, the black screen containing white text informing the participant where to stand on the next trial appeared.

Participants engaged in 10 familiarization trials after which the calibration of the mobile eyetracking system was checked. Participants then completed the test film in two blocks of 36 trials, lasting approximately nine minutes each. There was a five-minute break between the blocks in which the eye movement system calibration was checked again. The experiment was filmed using two HD cameras. One camera was positioned two meters behind the baseline and the other two meters to the right of the court, perpendicular to the service area. After the experiment, the verbal responses from the participants recorded on the video footage were individually checked against those originally hand notated. Reliability checks were carried out on $10 \%$ of the verbal response data using intra-observer (98\%) and inter-observer (91\%) agreement methods (Thomas, Nelson, \& Silverman, 1996).

\subsection{Data analysis}

Response accuracy was calculated as a score showing the number of correct verbal responses made by participants on the 72 trials. An independent $t$-test was used to compare overall response accuracy performance between the two groups in terms of the frequency of correct trials out of the 72 trials. Mean response accuracy scores for correct trials across the occlusion points were analyzed using a 2 Group (expert, novice) $\times 3$ occlusion points (shuttle-racket contact, $40 \mathrm{~ms}$ before, $40 \mathrm{~ms}$ after) ANOVA with repeated measures on the last factor. Separate one-way ANOVAs were used to examine the type of errors (depth, direction or both) made by the two groups.

Eye movement data were recorded at 25 frames per second. The video footage from the eye movement registration system was subjected to frame by frame analysis using Adobe Premier Pro Video Editing Software (Version CS 5, San Jose, USA). First, we conducted a standard visual search behavior analysis to enable comparison with previous research (e.g., Williams \& Elliott, 1999). Four gaze behaviors were initially calculated: number of fixations, fixation location, fixation duration, and duration of final fixation. A fixation was defined as when the participant's gaze remained within three degrees of visual angle of a location or moving object for a minimum duration of $120 \mathrm{~ms}$ (Vickers, 1996). Final fixation was defined as the last fixation on the screen prior to the video occluding. Separate independent $t$-tests were used to investigate eye movement patterns between the expert and novice groups for number of fixations, fixation duration, and duration of final fixation.

Second, in order to address our main hypotheses, we analyzed the visual fixation location data as a function of movement time and the response accuracy of the anticipation judgment. Initial inspection of the data revealed that each movement phase (preparation, execution) contained one visual fixation on average across participants. Therefore, visual fixation data were expressed as the frequency of trials in which a location was fixated separately for the movement phase in which it occurred. Visual fixation location categories were those body areas that differentiated short from long serves. Initial inspection of the fixation location data revealed that participants rarely, if at all, fixated the elbow or the shoulder. Therefore, even though these two areas differentiated serve-types, they were not analyzed separately in this analysis. Visual fixation location analysis was carried out on the kinematic locations at the racket and wrist, as well as the shuttle, with all other locations that did not discriminate between serve types being placed in a category called other, including the elbow and shoulder. The frequency of trials in which a location was fixated during the two phases (preparation, execution) of the movement were analyzed in separate 2 Group (expert, novice) $\times 2$ response accuracy (correct, incorrect) $\times 4$ location (racket, wrist, shuttle, other) ANOVAs with repeated measures on the last two factors. Any violations of the assumption of sphericity were corrected using the Greenhouse-Geisser method. Any significant interactions were analyzed using Tukey's Honestly Significant Difference. Bonferroni comparisons were used for main effects involving more than two variables. For all statistical tests, the alpha level for significance was .05 . 


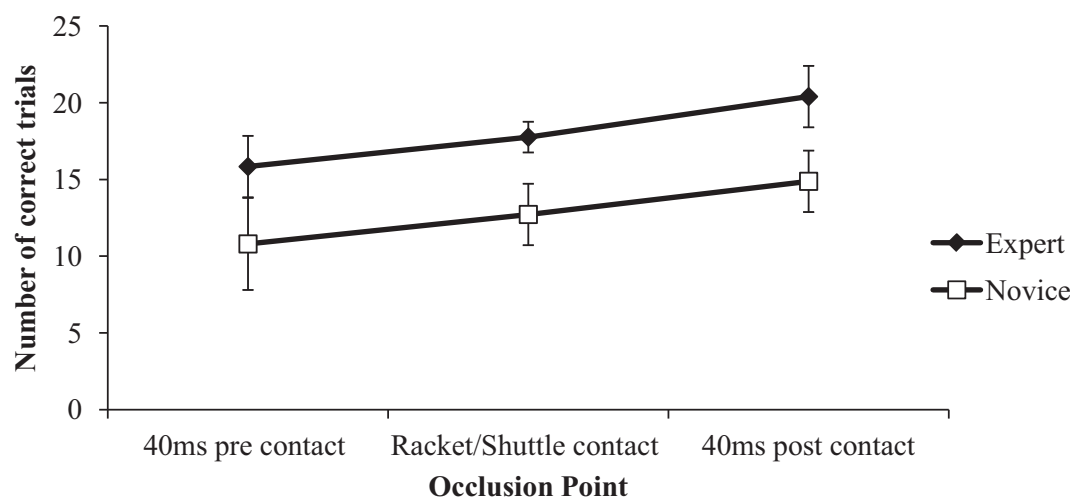

Fig. 1. Mean (SD) response accuracy of the expert and novice groups on the temporal occlusion anticipation test.

\section{Results}

Fig. 1 shows the response accuracy scores for both groups on the anticipation test. Response accuracy was significantly higher for the expert $(M=54$ correct trials out of 72 trials, $S D=3)$ when compared to the novice players $(M=39$ correct trials, $\mathrm{SD}=5), t(14)=7.46, p<.01$. The group (expert, novice) $\times$ occlusion points (shuttle-racket contact, $40 \mathrm{~ms}$ before, $40 \mathrm{~ms}$ after) ANOVA revealed a significant main effect for group, $F(1,14)=59.76, p<.01$. There was a significant main effect for occlusion point, $F(2,14)=30.49, p<.01$, with response accuracy being significantly higher in the $40 \mathrm{~ms}$ post racket/shuttle contact occlusion point $(M=18$ correct trials out of 24 trials, $S D=0.21$ ) compared to both the racket/shuttle contact occlusion point $(M=15$ correct trials, $\mathrm{SD}=1.12)$ and the $40 \mathrm{~ms}$ pre racket/shuttle contact occlusion point $(M=13$ correct trials, $S D=0.32$ ). There were significantly more correct trials in the racket/shuttle contact occlusion period compared to the $40 \mathrm{~ms}$ pre racket/shuttle contact occlusion points.

Response errors were made by incorrectly stating shot depth, shot direction, or shot depth and direction combined. One way ANOVA revealed novice players made significantly more direction errors $(M=17$ incorrect trials out of $34, \mathrm{SD}=4)$ compared to depth errors $(M=7$ incorrect trials out of 34 , $\mathrm{SD}=1)$ and combined depth and direction errors $(M=10$ incorrect trials out of $34, \mathrm{SD}=1), F(2,14)$ $=30.45, p<.01$. A separate one-way ANOVA showed skilled players made significantly more direction errors ( $M=9$ incorrect trials out of $18, \mathrm{SD}=1)$ when compared to depth errors $(M=4$ incorrect trials out of $18, S D=1)$ and combined depth and direction errors $(M=5$ incorrect trials out of $18, S D=1), F$ $(2,14)=44.81, p<.01$. Players who made direction errors may plausibly have been able to return the shuttlecock in a real-game due to their correct depth response placing them within the vicinity of it, whereas those who made errors on depth would be less likely to return it, if at all.

\subsection{Visual search behavior}

The overall number of visual fixations during trials in the temporal occlusion test did not differentiate the expert $(M=2.02$ fixations per trial, $\mathrm{SD}=0.68)$ and novice players $(M=1.88$ fixations per trial, $\mathrm{SD}=0.26), t(14)=-.57, p=.58$. Mean duration of fixation for the expert players $(M=1154 \mathrm{~ms}$ per trial, $\mathrm{SD}=169)$ was significantly longer compared to the novice players $(M=968 \mathrm{~ms}$ per trial, $\mathrm{SD}=103), t(14)=-2.64, p<.01$. The final fixation for the expert group $(M=1700 \mathrm{~ms}, \mathrm{SD}=132)$ was significantly longer compared to the novice players $(M=1262 \mathrm{~ms}, \mathrm{SD}=221), t(14)=-2.91, p<.01$.

The group (expert, novice) $\times$ response accuracy (correct, incorrect) $\times$ location (racket, wrist, shuttle, other) ANOVA on the visual fixation locations in the preparation phase revealed no significant main effects or interactions. Table 4 shows the statistical results for the same ANOVA on the fixation location data in the execution phase of the movement, while Fig. 2 shows the fixation locations for 
Table 4

The Results of the ANOVA for fixation locations during the execution phase of the movement for the expert and novice group on successful and unsuccessful performance.

\begin{tabular}{lllll}
\hline Variable & MS & MSE & $F$ & Cohen's effect size \\
\hline Response accuracy & 800 & 9.32 & $85.82^{* * *}$ & 0.86 \\
Group & - & - & - & - \\
Response accuracy $\times$ group & 480.50 & 9.32 & $51.55^{* *}$ & 0.79 \\
Location & 2056.33 & 14.99 & $137.14^{* *}$ & 0.91 \\
Location $\times$ group & 260.08 & 14.99 & $17.35^{* *}$ & 0.55 \\
Response accuracy $\times$ location & 697.92 & 13.60 & $51.34^{* *}$ & 0.79 \\
Response accuracy $\times$ location $\times$ group & 85.08 & 13.60 & $6.26^{* *}$ & 0.31 \\
\hline
\end{tabular}

** $P<0.01$.

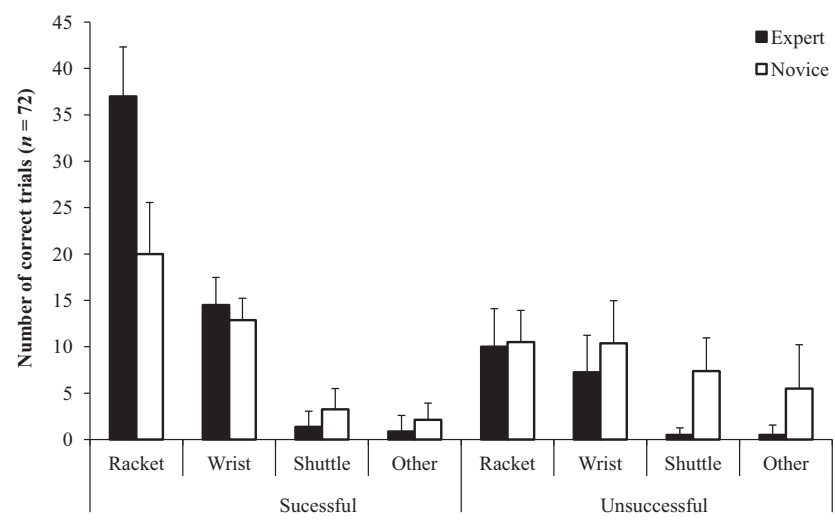

Fig. 2. Mean (SD) visual fixation locations for the expert and novice groups in the execution phase of the movement.

both groups in the execution phase. A significant main effect for fixation location showed participants fixated vision on the racket in more trials compared to the wrist, shuttle or other location. Moreover, the wrist was fixated in more trials compared to the shuttle and other location.

There was a significant three-way response accuracy $\times$ location $\times$ group interaction that explained the interactions in the data. Post hoc showed that the expert players fixated the racket on more of their 54 correct trials $(M=37$ out of 54 correct trials, $S D=2.21)$ compared to the novice players on their 39 correct trials $(M=20$ out of 39 correct trials, $S D=2.11)$ and that they fixated on the racket in more correct trials compared to all other locations for both groups across all trials $(p<.05)$. There were no significant differences in the amount of incorrect trials in which the racket was fixated between the expert $(M=10$ trials out of an average of 18 incorrect trials, $S D=1.32)$ and novice group $(M=11$ trials out of an average of 33 incorrect trials, $S D=1.02)$. However, both groups fixated the racket significantly more on correct trials compared to incorrect trials $(p<.05)$, with the number of incorrect trials in which the racket was fixated being relatively low. For the wrist location, there were no between-group differences in the number of correct trials in which fixations upon the wrist occurred for the expert ( $M=14$ trials out of an average of 54 correct trials, $S D=1.43$ ) and novice players $(M=13$ trials out of an average of 39 correct trials, $S D=1.06)$. However, the novice players fixated the wrist on more incorrect trials ( $M=10$ out of an average of 33 incorrect trials, $S D=2.32$ ) compared to the expert players ( $M=7$ out of an average of 18 incorrect trials, $S D=2.11$ ). Both groups fixated the wrist in more correct compared to incorrect trials $(p<.05)$.

For the shuttle location, the novice players fixated the shuttle in more correct ( $M=3$ out of 39 correct trials, $\mathrm{SD}=1.01)$ and incorrect trials $(M=7$ out of 33 incorrect trials, $\mathrm{SD}=1.21)$ compared to the expert players. However, the number of correct trials in which the shuttle was fixated was very low and was significantly lower than for the racket and wrist $(p<.05)$, while for the novice players it 
occurred on significantly more incorrect compared to correct trials $(p<.05)$. For the other locations, the novice players fixated them in more incorrect trials $(M=6$ out of 33 incorrect trials, SD $=1.32$ ) compared to the expert players, but there were no between-group differences in fixations on other locations in correct trials, with the number of trials in which this occurred being very low. Moreover, the novice group fixated the other location more during incorrect trials compared to correct trials, whereas the expert group did not.

\section{General discussion}

We examined the kinematic differences between long and short badminton serves and whether athletes fixate vision upon the discriminating kinematic information during successful anticipation responses. A comprehensive kinematic analysis demonstrated when and where the discriminating kinematic differences occurred between long and short serves. Subsequently, expert and novice participants completed a temporal occlusion anticipation test while wearing a mobile eye movement registration system. As predicted, the expert participants were significantly more accurate at anticipation on the temporal occlusion test compared to the novice participants. Findings support previous research showing that expert athletes are better than novice athletes at anticipating upcoming actions (Abernethy, Gill, Parks, \& Packer, 2001; Dicks, Button, \& Davids, 2010; Gabbett, Rubinoff, Thorburn, \& Farrow, 2007; Muller, Abernethy, \& Farrow, 2006; Savelsbergh et al., 2002; Williams et al., 2002), especially earlier in the execution of the action (Abernethy, 1990; Williams et al., 2002). The expert group have a significantly greater amount of domain specific experience, thus enabling them to combine the current environmental situation with those previous experiences to aid in response selection (Causer et al., 2012), whereas the novices do not.

The kinematic differences between long and short serves occurred in the arm, wrist, and racket regions during the execution phase of the movement, whereas there were no kinematic differences between serves in the preparation phase. During the temporal occlusion test, it was expected that the expert players would fixate vision upon the differentiating kinematic locations when this information was available more frequently compared to the novice players. In accordance with this prediction, during the execution phase of the movement, the expert players fixated the racket in more trials and in more correct trials compared to the novice players and compared to any other location. Moreover, both groups fixated upon the discriminating location of the wrist during the execution phase more so than the shuttle and other locations and it was fixated more in correct compared to incorrect trials. However, although the elbow and shoulder were discriminating kinematic locations, participants did not fixate vision upon them, demonstrating that the most relevant cues regarding shot-type were emanating from more distal kinematic locations (i.e., the wrist and the racket). The visual fixation data supports previous research on badminton shots showing that fixations during anticipation judgments are located on specific distal areas of the body, such as arm, wrist, and racket (Abernethy \& Russell, 1987). It contradicts previous research in tennis showing fixation locations tend to be distributed across a number of central regions of the body (e.g., head, trunk in Ward et al., 2002; Williams et al., 2002). Therefore, it seems the contradictory results between researchers investigating the kinematic differences between actions (e.g., Bourne et al., 2011) and those examining the kinematic information that observers fixate vision upon (e.g., Williams et al., 2002) are a product of examining these processes in isolation from one another.

In contrast, the novice players fixated vision upon the shuttle and other locations, which did not differentiate serve-type, in more trials and in more incorrect trials compared to the expert players. These data agree with previous research by showing that less-skilled players fixate vision upon locations on the opponent's body in which no between-shot differences occur (Savelsbergh et al., 2002, 2005). It was predicted that both groups would be more accurate at anticipation when fixating on the discriminating kinematic locations compared to when they fixated other areas of the display. In support of this prediction, players fixated the racket and wrist in significantly more correct compared to incorrect trials and compared to the shuttle and other locations. Data supports Savelsbergh et al. (2005) who found that the location upon which vision is fixated was a key factor in distinguishing between successful and unsuccessful anticipation performance. In the current study, participants 
fixated on the wrist and racket in more incorrect trials compared to the shuttle and other locations, albeit the number of trials in which this occurred was relatively low $(n=10$ trials per group per location). These data indicate some incorrect anticipatory judgments occurred when athletes fixated on the correct kinematic differences between actions, but failed to recognize or use this information correctly. However, novice players also made more incorrect judgments compared to expert players when they fixated on the shuttle and other locations, which did not differentiate serve-types. Findings extend previous research by showing that novice players made anticipatory errors when they fixated on the incorrect kinematic locations, whereas expert player did not fixate on this information.

It was expected that the kinematic differences between serve-types would emerge or disappear as the movement unfolded across time. The kinematic data supported this prediction as differences between serve-types were found to emerge only in the final execution phase of the movement. During the preparation phase of the badminton serve there were no differences between long and short serves for distance covered at all locations, except for the right elbow. Previously, researchers examining the kinematic cues used during anticipation (Abernethy \& Russell, 1987; Abernethy et al., 2008) have suggested this information is available throughout the movement of the opponent, whereas our data show it is not. As predicted, during the execution phase of the movement, the kinematic locations of the arm, wrist, and racket discriminated between the two serve-types. Findings provide support and explanation for researchers who showed that athletes change the areas they fixate upon across the ongoing movement (Abernethy \& Russell, 1987; Kim \& Lee, 2006; Savelsbergh et al., 2002). In our study, the amount and type of kinematic information emanating from different areas of the opponent's body changed across the movement.

The standard visual search behavior analysis used by other researchers (Williams \& Elliott, 1999) revealed no between-group differences for the number of fixations per trial. These data contradict previous research showing that expert performers in relatively closed tasks tend to make fewer fixations when compared to novice players (Abernethy, 1990; Poliszczuk \& Mosakowsk, 2009; Savelsbergh et al., 2002). These data may be due to the badminton serve and the relatively short movement time for each phase, with the execution phase being only $1900 \mathrm{~ms}$ in duration. However, the expert players had significantly longer fixation durations and final fixations compared to the novice players, supporting previous research. Their average final fixation of $1700 \mathrm{~ms}$ corresponded well with the duration of the execution phase, which was an average of $1900 \mathrm{~ms}$. Data support previous research on moderately consistent and controlled tasks (Abernethy et al., 2008; Williams et al., 2002) showing fixations of a longer duration are indicative of expert performance. It is possible that the longer fixation duration allowed the expert group more time to extract information from the kinematic cues emanating from the opponent's body.

The data show for the first time that the visual search behavior of the athlete anticipating an opponent's action is dependent on the kinematics of that action and that these two variables should not be examined in isolation. Findings contradict the strategy of researchers who have investigated the kinematic differences between movements in isolation (e.g., Huys et al., 2008) and those who have separately examined the kinematic information that observers fixate upon during anticipation (Williams et al., 2002). In the current study, we have shown that the visual search behavior of the observer is linked to, and interacts with, the kinematics of the movement being observed. The kinematic differences between the long and short badminton serves used in this study occurred at specific and few distal locations in the arm, wrist, and racket. We have also shown that expert players fixate vision upon these kinematic differences between actions more so compared to novice players and that fixating vision on these differences is associated with superior anticipatory judgments. In future researchers examining anticipation should consider measuring visual search behaviors and the kinematics of the action being observed. Moreover, perceptual training programmes aiming to develop more efficient anticipation performance should seek to match visual search patterns to the observed movement. It may be that the temporal constraints of anticipating a badminton serve are less severe compared to some other tasks, such as a smash in badminton or tennis. In this study, coaches identified the serve in a doubles match as a key shot that required anticipation as the majority of points won in badminton doubles are won by the pair who gain the attack on the second shot/return of serve (Badminton Association of England, Level 1: Assistant coach training manual, 2005). Given that not all shots in these sports require anticipation (Triolet, Benguigui, Le Runigo, \& Williams, 2013) in future 
researchers should seek to objectively identify shots that do. In the current study, the kinematic analysis and the test film footage were collected in separate sessions. The kinematic data collection involved the court being encircled by four Motion Capture cameras, as well as the server wearing 28 reflective markers. We felt that a separate session was required to collect the video footage in order to maintain a level of ecological validity and ensure that participants did not attend to the markers instead of engage in their usual visual strategy. In future researchers should seek to collect both sets of data in one session using methods that are less invasive and ecologically limiting (i.e., SimiMotion, Germany). The expert group in this study may have had previous experience of playing with or against the servers used to generate video footage, which was an unavoidable by-product of the small expert population.

In summary, we examined for the first time in the literature the coupling between the kinematics differentiating opponent actions and the related visual search behavior of expert and novice badminton players aiming to anticipate these actions. Long and short badminton serves were differentiated for distance traveled and peak acceleration at the kinematic locations of the arm, wrist and racket. Expert players fixated vision on the locations that were discriminating between serve-types for a longer duration and more frequently when compared to the novice players. The expert players' visual search strategies resulted in more accurate anticipation when compared to the novice players. These data extend previous literature by showing that expert players' visual search behavior is inextricably linked to the kinematics of the opponents' actions being observed and that this is an essential part of successful anticipatory judgements. Data has implications for applied practitioners in badminton and, possibly, other racket sports attempting to develop perceptual-cognitive skills through providing prescriptive information relating to the critical cues that aid anticipatory judgements.

\section{Acknowledgements}

The lead author was funded by the English Institute of Sport during this research. There are no conflicts of interest arising from this research.

\section{References}

Abernethy, B. (1990). Anticipation in squash: Differences in advance cue utilization between expert and novice players. Journal of Sport Sciences, 8, 17-34.

Abernethy, B., Gill, D. P., Parks, S. L., \& Packer, S. T. (2001). Expertise and the perception of kinematic and situational probability information. Perception, 30, 233-252.

Abernethy, B., \& Russell, D. G. (1987). The relationship between expertise and visual-search strategy in a racquet sport. Human Movement Science, 6, 283-319.

Abernethy, B., Zawi, K., \& Jackson, R. C. (2008). Expertise and attunement to kinematic constraints. Perception, 37, $931-948$.

Badminton Association of England (2005). Level 1: Assistant coach training manual. Milton Keynes, UK: BAE.

Bourne, M., Bennett, S. J., Hayes, S. J., \& Williams, A. M. (2011). The dynamical structure of handball penalty shots as a function of target location. Human Movement Science, 30, 40-55.

Causer, J., Janelle, C. M., Vickers, J. N., \& Williams, A. M. (2012). Perceptual expertise: What can be trained? In N. J. Hodges \& A. M. Williams (Eds.), Skill acquisition in sport: Research, theory and practice (pp. 306-324). London: Routledge.

Dempsey, A. R., Lloyd, D. G., Elliott, B. C., Steele, J. R., \& Munro, B. J. (2009). Changing sidestep cutting technique reduces knee valgus loading. American Journal of Sport Medicine, 37, 2194-2200.

Dicks, M., Button, C., \& Davids, K. (2010). Examination of gaze behaviors under in situ and video simulation task constraints reveals differences in information pickup for perception and action. Attention, Perception \& Psychophysics, 72, 706-720.

Gabbett, T., Rubinoff, M., Thorburn, L., \& Farrow, D. (2007). Testing and training anticipation skills in softball fielders. International Journal of Sports Science and Coaching, 2, 15-24.

Huys, R., Smeeton, N. J., Hodges, N. J., Beek, P. J., \& Williams, A. M. (2008). On the dynamic information underlying visual anticipation skill. Perception \&' Psychophysics, 70, 1217-1234.

Kim, S., \& Lee, S. (2006). Gaze behaviour of elite soccer goalkeeper in successful penalty kick defence. International Journal of Applied Sport Science, 18, 96-110.

Lees, A. (2002). Technique analysis in sport: A critical review. Journal of Sports Sciences, 20, 813-828.

Muller, S., Abernethy, B., \& Farrow, D. (2006). How do world-class cricket batsmen anticipate a bowler's intention? Quarterly Journal of Experimental Psychology, 59, 2162-2186.

Poliszczuk, T., \& Mosakowsk, M. (2009). Inter-reactions of peripheral perception and ability of time-movement anticipation in high class competitive badminton players. Studies in Physical Culture and Tourism, 16, 259-265.

Ripoll, H., Kerlirzin, Y., Stein, J. F., \& Reine, B. (1995). Analysis of information processing, decision making, and visual strategies in complex problem solving sport situations. Human Movement Science, 14, 325-349.

Savelsbergh, G. J., Van der Kamp, J., Williams, A. M., \& Ward, P. (2005). Anticipation and visual search behaviour in expert soccer goalkeepers. Ergonomics, 48, 1686-1697. 
Savelsbergh, G. J., Williams, A. M., Van der Kamp, J., \& Ward, P. (2002). Visual search, anticipation and expertise in soccer goalkeepers. Journal of Sport Sciences, 20, 279-287.

Thomas, J. R., Nelson, J. K., \& Silverman, S. J. (1996). Research Methods in Physical Activity. Champaign, IL: HumanKinematics.

Triolet, C., Benguigui, N., Le Runigo, C., \& Williams, A. M. (2013). Quantifying the nature of anticipation in professional tennis. Journal of Sports Sciences, 31, 820-830.

Vickers, J. N. (1996). Visual control when aiming at a far target. Journal of Experimental Psychology, 22, 342-354.

Ward, P., Williams, A. M., \& Bennett, S. J. (2002). Visual search and biological motion perception in tennis. Research Quarterly for Exercise in Sport, 73, 107-112.

Williams, A. M., \& Elliott, D. (1999). Anxiety, expertise, and visual search strategy in karate. Journal of Sport E Exercise Psychology, $21,362-375$.

Williams, A. M., Ford, P. R., Eccles, D. W., \& Ward, P. (2011). Perceptual-cognitive expertise in sport and its acquisition: Implications for applied cognitive psychology. Applied Cognitive Psychology, 25, 432-442.

Williams, A. M., Ward, P., Knowles, J. M., \& Smeeton, N. J. (2002). Anticipation skill in a real-world task: measurement, training, and transfer in tennis. Journal Experimental Psychology: Applied, 8, 259-270. 